\title{
Successful pregnancy outcome in a rare case of complete situs inversus in a primigravida
}

\section{Radhika BH*, Harshini V, Priyanka Jogi, Renuka Ramaiah}

\begin{abstract}
Department of Obstetrics and Gynecology, Employee's State Insurance Corporation Post Graduate Institute of Medical Sciences and Research, Bangalore, Karnataka, India
\end{abstract}

Received: 7 April 2015

Accepted: 6 June 2015

\section{*Correspondence: \\ Radhika BH, \\ E-mail: radsgowda85@gmail.com}

Copyright: $\odot$ the author(s), publisher and licensee Medip Academy. This is an open-access article distributed under the terms of the Creative Commons Attribution Non-Commercial License, which permits unrestricted non-commercial use, distribution, and reproduction in any medium, provided the original work is properly cited.

\begin{abstract}
Complete situs inversus is a rare syndrome, of autosomal recessive inheritance, resulting from inversion of rotation of the cardiac tube during embryogenesis. It occurs in 1 in 10,000 births. We report here an interesting case where situs inversus was diagnosed during pregnancy followed by uneventful course of pregnancy. A 28 year old primigravida, presented to our hospital for first antenatal visit at 16 weeks of gestation. On physical examination she was found to have her cardiac apex on the right side in right 5th intercostal space. Presence of dextrocardia prompted a thorough physical examination, with other findings raising suspicion of total situs inversus. Confirmation of situs inversus was done by ultrasound imaging of abdomen and transthoracic echocardiography. Her obstetric ultrasonographic examination was unremarkable and showed a single alive intrauterine gestation 14 weeks gestation. She was regularly followed up \& her pregnancy course was uneventful. She was admitted at term gestation, with premature rupture of membranes. An attempt to induce labour was done with four doses of oral misoprostol. Eventually she underwent emergency cesarean section due to failure of induction and an alive term female baby of weight $2.7 \mathrm{~kg}$ was delivered . Intra and post-operative period was uneventful. She was discharged on sixth post-operative day. Her six week postpartum follow up was uneventful. Situs inversus with pregnancy is a rare clinical entity. However, these patients are likely to have uneventful course during pregnancy.
\end{abstract}

Keywords: Complete situs inversus, Pregnancy

\section{INTRODUCTION}

It is first described by Aristotle in animals and Fabricius in humans. In general, each thoracic or abdomina ${ }^{1}$ structure is always located to one side or the other of the midline, as is the case with heart, aortic arch, stomach and spleen to the left, with liver and gallbladder to the right. ${ }^{1}$ This anatomic left-right relation of the asymmetric viscera is known as situs solitus. The disturbance in the establishment of left-right asymmetry results in situs inversus. Situs inversus can be either total or partial. Total situs inversus, also known as mirror image dextrocardia, is characterized by the heart on right side of the midline, while the liver and gallbladder are on left side. The exact etiology is unknown, but the autosomal recessive mode of inheritance has been speculated. ${ }^{1}$

\section{CASE REPORT}

A 28 year old primigravida, on her first antenatal visit, was found to have dextrocardia on physical examination due to palpation of cardiac apical impulse in right 5th intercostal space. Presence of dextrocardia prompted a thorough physical examination. Physical examination revealed pulse rate of $84 / \mathrm{min}$ with a pressure of $130 / 70$ $\mathrm{mm} \mathrm{Hg}$. There were no apparent spinal or neurological deformities. Auscultation revealed normal heart sounds heard in the right 5 th intercostal space. Percussion could not be done due to pregnant uterus. Ultrasound abdomen 
showed liver and gallbladder situated on the left side, spleen and heart situated on the right. ECG revealed right axis deviation, positive QRS complexes in a VR, negative QRS, $\mathrm{P}$ wave and $\mathrm{T}$ wave in lead I and reverse R-wave progression in the chest leads. Echocardiogram showed situs inversus dextrocardia, normal valves, chambers and normal LV function, right aortic arch and no shunt lesions. Foetal echocardiogram was normal. Patient was asymptomatic throughout the pregnancy. She was admitted at 38 weeks of gestation, due to premature rupture of membranes (PROM). An attempt to induce labour was made with four doses of vaginal misoprostol in view of PROM. Subsequently emergency LSCS was done under subarachnoid block, in view of PROM with failed induction and an alive term female baby of weight $2.7 \mathrm{~kg}$ was delivered. Intra and post-operative period was uneventful. She was discharged on sixth post-operative day. Initial investigations of baby with ultrasonography and echocardiography did not reveal any abnormality. Her six week postpartum follow up was normal.

\section{DISCUSSION}

Situs describes the position of the cardiac atria and viscera. Situs solitus is the normal position and situs inversus is the mirror image of situs solitus. Cardiac situs is determined by the atrial location. In situs inversus, the morphologic right atrium is on the left, and left atrium is on the right. This reverse phenomenon applies to pulmonary anatomy also, hence the left lung may have 3 lobes and the right lung may have 2 lobes. In addition, the liver and gallbladder are located on the left, whereas the spleen and stomach are located on the right. ${ }^{2}$

Situs inversus, is an rare anomaly, with overall frequency of $1 / 10,000$ live births. ${ }^{3}$ Complete situs inversus is an even rarer syndrome and is of autosomal recessive inheritance. ${ }^{1,3}$ It is equally distributed among both the sexes. And there is no difference between races. Recent studies suggest the left right asymmetry defects are likely to be due to genetic abnormalities. ${ }^{3}$

Very few cases of maternal situs inversus during pregnancy have been reported so far in the literature. ${ }^{1,4}$

Situs inversus can either be partial or total. Situs inversus further classified into situs inversus with levocardia or situs inversus with dextrocardia. ${ }^{3}$ In situs inversus with dextrocardia (situs inversus totalis) cardiac position, atrial chambers and abdominal viscera are the mirror images of the normal anatomy. Most patients with situs inversus have dextrocardia as their only cardiac manifestation. ${ }^{4}$

The incidence of congenital heart disease (atrial or ventricular septal defects, tetralogy of Fallots, pulmonary artery hypoplasia)in situs inversus with dextrocardia, is only $3-5 \%$. Of these patients, $80 \%$ have a right-sided aortic arch. ${ }^{2}$ In contrast Situs inversus with levocardia is rare, and it is almost always associated with congenital heart disease. Congenital anomalies such as kartagener syndrome, asplenia, polysplenia are known to occur in situs inversus with levocardia. Kartagener syndrome is chracterised by bronchiectasis, sinusitis and situs inversus and affects $20 \%$ of patients with situs inversus; only $50 \%$ of patients with Kartagener syndrome have situs inversus. $^{2}$ However, our patient did not have any symptomatology suspicious for Kartagener syndrome.

Diagnosis is based on clinical examination and complementary diagnostic techniques such as electrocardiograms, echocardiography, radiography, ultrasonography, CT scan, MRI, barium studies. ${ }^{1}$ In our study, we diagnosed the condition by ultrasonography and echocardiography.

Prognosis for patients with isolated dextrocardia depends on the underlying structural and functional defects since it is associated with high incidence of severe congenital anomalies such as single ventricle, transposition of the great vessels, complete atrioventricular septal defects, anomalous pulmonary venous return, tricuspid valve atresia, pulmonary artery atresia, or hypoplasia and coarctation of aorta. Life expectancy in patients with situs inversus totalis with dextrocardia, is the same as for the general population. ${ }^{3}$ Our patient did not have any associated congenital abnormality and was asymptomatic throughout pregnancy.

Situs inversus usually remains undiagnosed, unless it is diagnosed incidentally, while investigating other associated elements. ${ }^{3}$ Our patient had dextrocardia and the diagnosis of situs inversus totalis was made when she was evaluated for dextrocardia. In our patient, pregnancy and post partum period was uneventful.

\section{CONCLUSION}

Situs inversus usually remains undiagnosed, unless it is diagnosed incidentally. Situs inversus with pregnancy is a rare clinical entity. However, these patients in the absence of other associated syndromic anomalies are likely to have uneventful course during pregnancy.

\section{Funding: No funding sources \\ Conflict of interest: None declared \\ Ethical approval: Not required}

\section{REFERENCES}

1. Tayeb M, Khan FM, Rouf F. Situs inversus totalis with perforated duodenal ulcer; a case report: Journal of medical case reports. 2011;5:279.

2. Wilhelm A, Karani J. Situs inversus imaging. 2010 [updated October 2013].Available from:http://www.emedicine.medscape.com/article/41 3679-overview.

3. Marta MJ, Falcão LM, Saavedra JA, Ravara L. A case of complete situs inversus. Rev Port Cardiol. 2003;22(1):91-104. 
4. Said SAM, Veerbeek A, Van Der Weiken LR. Dextrocardia, situs inversus and severe mitral stenosis in a pregnant woman:successful closed commissurotomy: European heart journal. 1991;12(7):825-8.
Cite this article as: Radhika BH, Vana $\mathrm{H}$, Jogi $\mathrm{P}$, Ramaiah R. Successful pregnancy outcome in a rare case of complete situs inversus in a primigravida. Int $\mathbf{J}$ Reprod Contracept Obstet Gynecol 2015;4:1203-5. 